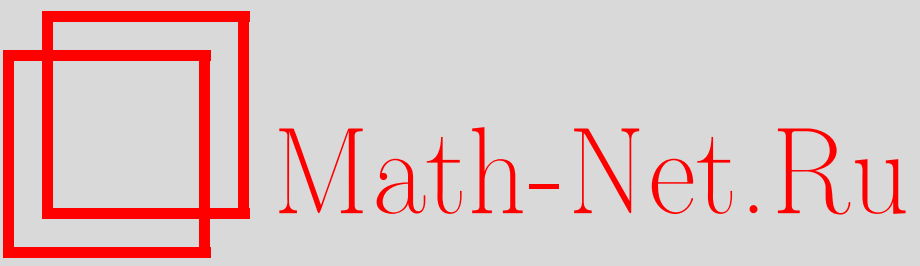

Г. М. Жислин, Локализация существенного спектра операторов энергии квантовых систем с невозрастающим магнитным полем, ТМФ, 1996, том 107, номер 3, 372-387

DOI: https://doi.org/10.4213/tmf1163

Использование Общероссийского математического портала Math-Net.Ru подразумевает, что вы прочитали и согласны с пользовательским соглашением

http://www.mathnet.ru/rus/agreement

Параметры загрузки:

IP : 18.207 .199 .55

26 апреля 2023 г., 14:05:24 


\author{
ТЕОРЕТИЧЕСКАЯ \\ И МАТЕМАТИЧЕСКАЯ \\ ФИЗИКА \\ Том 107, № 3 \\ июнь, 1996
}

\title{
Г.М. Жислин
}

\section{ЛОКАЛИЗАЦИЯ СУШЕСТВЕННОГО СПЕКТРА ОПЕРАТОРОВ ЭНЕРГИИ КВАНТОВЫХ СИСТЕМ С НЕВОЗРАСТАЮШИМ МАГНИТНЫМ ПОЛЕМ}

\footnotetext{
Теорема о локализации существенного спектра доказывается для не нейтральных
} многочастичных систем в невозрастающих магнитных полях, имеющих $S O(2)$-симметрию.

\section{ВВЕДЕНИЕ}

В этой статье мы продолжаем изучение существенного спектра $n$-частичного гамильтониана с магнитным полем, начатое в $[1,2]$. Как и ранее, мы рассматриваем только магнитные поля, имеющие направление оси $x_{3}$ и не зависящие от $x_{3}$. Главная трудность исследования систем в таких магнитных полях состоит в невозможности отделить движение центра масс (ц.м.) системы в плоскости $x_{1}, x_{2}$ : это невозможно даже для однородного магнитного поля, если частицы не тождественны[3]. Чтобы преодолеть эту трудность, мы рассматривали в $[1,2]$ поля, которые стремятся к $+\infty$ во всех направлениях плоскости $x_{1}, x_{2}$ и, следовательно, не разрешают движение ц.м. к бесконечности в этой плоскости. Для таких полей теоремы о локализации существенного спектра были доказаны в $[1,2]$ для широкого класса квантовых систем, включая произвольные атомы и молекулы.

Чтобы получить локализационную теорему для невозрастающих (в плоскости $x_{1}$, $\left.x_{2}\right)$ полей мы предложили в [1] использовать $S O(2)$-симметрию системы, т.е. изучать спектр гамильтонианов на подпространствах функций фиксированного типа $m$ $S O(2)$-симметрии. Однако для таких полей результаты $[1,2]$ были установлены только при условии, что заряды $e_{j}$ всех частищ системы имеют один и тот же знак (к несчастью, по вине авторов это условие было пропушено в $[4,5]$, где наши результаты для невозрастающих полей были опубликованы без доказательств). Позднее для не нейтральных систем это условие было снято, но только для однородного магнитного поля и для систем без нейтральных частиц [6].

В этой статье мы возврашаемся к исследованию операторов энергии квантовых систем в невозрастающих магнитных полях для фиксированного типа $m S O(2)$-симметрии. Мы доказываем общее утверждение о локализации сушественного спектра таких систем и даем достаточные условия его применимости (теоремы 2.1, 2.2). Эти условия для магнитного поля означают, что магнитный вектор-потенциал не может вести себя 
очень "нерегулярно" на бесконечности и что допустимая "нерегулярность" зависит от отношения суммарного заряда системы к сумме модулей зарядов всех частиц системы (cм. (2.5)).

Доказанные теоремы обнаруживают сушественный спектр для широкого класса заряженных систем (атомные и молекулярные ионы; системы, содержащие нейтральные частицы) в невозрастающем магнитном поле, в том числе и в однородном магнитном поле. Для нейтральных систем наши результаты не применимы, и результат [3] для однородного магнитного поля является единственным.

Наш метод геометрический. Так же как и метод [1] , он основан на разбиении конфигурационного пространства на области, которые соответствуют возможным разбиениям системы. Но здесь (в отличие от [1]) мы применяем различные типы разбиений для различных частей конфигурационного пространства. Сначала мы делаем разбиение в направлении третьей оси для больших значений $\sum_{i} x_{i 3}^{2}$, а после этого - разбиение в плоскости $x_{1}, x_{2}$ (для ограниченных значений $\sum_{i} x_{i 3}^{2}$ ). Особенно важным является тот факт, что разбиение в плоскости $x_{1}, x_{2}$ учитьвает возможность движения ц.м. системы к бесконечности (в этой плоскости). Для простоты в этой статье мы не учитываем перестановочную симметрию, т.к. это может быть сделано аналогично [1] .

\section{1. ОПРЕДЕЛЕНИЯ}

1.1. Пусть $Z_{1}=(1,2, \ldots, n)$ - квантовая система $n$ частиц с зарядами $e_{j}$, массами $k_{j}$ и координатами $r_{j}=\left(\rho_{j}, x_{j 3}\right), \rho_{j}=\left(x_{j 1}, x_{j 2}\right)$ во внешнем магнитном поле с магнитным вектор-потенциалом

$$
\mathcal{B}=\left(\mathcal{B}_{1}, \ldots, \mathcal{B}_{n}\right), \quad \mathcal{B}_{j}=\left(\mathcal{B}_{j \perp}, 0\right), \quad \mathcal{B}_{j \perp}=B\left(\left|\rho_{j}\right|\right)\left(-x_{j 2}, x_{j 1}\right)
$$

$V_{s t}\left(\left|r_{s t}\right|\right)=V_{t s}\left(\left|r_{t s}\right|\right)-$ потенциалы взаимодействия частиц $s, t$ между собой, $r_{s t}=$ $r_{t}-r_{s}$,

$$
\begin{gathered}
M=\sum_{i=1}^{n} k_{i}, \quad r=\left(r_{1}, \ldots, r_{n}\right), \quad \rho=\left(\rho_{1}, \ldots, \rho_{n}\right), \quad X_{3}=\left(x_{13}, \ldots, x_{n 3}\right), \\
R^{3 n}=\{r\}, \quad R^{2 n}=\{\rho\}, \\
R_{03}=\left\{r \mid r \in R^{3 n}, \quad \rho_{j}=(0,0) \quad j=1,2, \ldots, n, \quad \sum_{i=1}^{n} k_{i} x_{i 3}=0\right\} \\
R_{0 \perp}=\left\{r \mid r \in R^{3 n}, x_{j 3}=0 \quad j=1, \ldots, n\right\}, \quad R_{0}=R_{0 \perp} \oplus R_{03},
\end{gathered}
$$

$R_{0}$ - пространство относительного движения системы $Z_{1}$ в направлении третьей оси.

Введем в $R^{3 n}$ новое скалярное произведение и норму. Пусть

$$
\widetilde{r}=\left(\widetilde{r}_{1}, \ldots, \widetilde{r}_{n}\right), \quad(r, \widetilde{r})_{1}=\sum_{i=1}^{n}\left[\left(\rho_{i}, \widetilde{\rho}_{i}\right)_{R^{2}}+k_{i} x_{i 3} \widetilde{x}_{i 3} M^{-1}\right], \quad|r|_{1}=(r, r)_{1}^{1 / 2}
$$

и $P_{0}$ - проектор ${ }^{1)}$ на пространство $R_{0}$. Тогда $q=\left(q_{1}, \ldots, q_{n}\right) \equiv P_{0} r$ суть координаты в $R_{0}, q_{j}=\left(\rho_{j}, q_{j 3}\right), q_{j 3}=x_{j 3}-M^{-1} \sum_{s=1}^{n} k_{s} x_{s 3}, r_{s t}=q_{t}-q_{s}$.

\footnotetext{
${ }^{1)}$ Все проекторы в п. 1.1-1.3 суть проекторы в смысле $(\cdot, \cdot)_{1}$.
} 
Пусть

$$
\begin{gathered}
\nabla_{t \perp}=\left(\frac{\partial}{\partial x_{t 1}}, \frac{\partial}{\partial x_{t 2}}\right), \quad \nabla_{t 3}^{\circ}=k_{t}^{-1} \frac{\partial}{\partial q_{t 3}}-M^{-1} \sum_{s=1}^{n} \frac{\partial}{\partial q_{s 3}}, \\
T_{t}=k_{t}^{-1}\left(i \nabla_{t \perp}+e_{t} \mathcal{B}_{t \perp}\right)^{2}, \quad T_{t 3}^{\circ}=-k_{t} \nabla_{t 3}^{\circ}, \\
A=\sum_{t=1}^{n} T_{t}, \quad T_{3}^{\circ}=\sum_{t=1}^{n} T_{t 3}^{\circ}, \quad V(q)=\frac{1}{2} \sum_{s, t ; s \neq t} V_{s t}\left(\left|q_{s t}\right|\right) .
\end{gathered}
$$

Тогда оператор энергии системы $Z_{1}$ (после отделения движения ц.м. в направлении оси $x_{3}$ ) может быть записан в виде

$$
H_{0}=A+T_{3}^{\circ}+V(q),
$$

где все операторы зависят только от $q$.

Мы предполагаем, что функции $V_{s t}\left(\left|r_{1}\right|\right)$ и $B\left(\left|\rho_{1}\right|\right)$ вешественны,

$$
V_{s t}\left(\left|r_{1}\right|\right) \in \mathcal{L}_{2, \text { loc }}\left(R^{3}\right), \quad s, t=1, \ldots, n, \quad\left|\rho_{1}\right| B\left(\left|\rho_{1}\right|\right) \in \mathcal{L}_{4, \text { loc }}\left(R^{2}\right)
$$

и

$$
\lim _{t \rightarrow+\infty} V_{s p}(t)=0
$$

1.2. Для произвольной системы $\mathcal{D}=\left(i_{1}, \ldots, i_{p}\right) \subseteq Z_{1}$ мы полагаем

$$
\begin{gathered}
R[\mathcal{D}]=\left\{r \mid r \in R^{3 n}, r_{i}=(0,0,0), i \bar{\in} \mathcal{D}\right\}, \quad R_{0 \perp}[\mathcal{D}]=\left\{r \mid r \in R[\mathcal{D}], x_{t 3}=0, t \in \mathcal{D}\right\}, \\
R_{03}[\mathcal{D}]=\left\{r \mid r \in R[\mathcal{D}], \rho_{t}=(0,0), t \in \mathcal{D}, \sum_{t \in \mathcal{D}} k_{t} x_{t 3}=0\right\}, \quad R_{0}[\mathcal{D}]=R_{0 \perp}[\mathcal{D}] \oplus R_{03}[\mathcal{D}] .
\end{gathered}
$$

Ясно, что $R_{0}[\mathcal{D}]$ есть пространство относительного движения кластера $\mathcal{D}$. Пусть $P_{0}[\mathcal{D}]-$ проектор в $R[\mathcal{D}]$ на подпространство $R_{0}[\mathcal{D}]$. Тогда

$$
\begin{aligned}
q[\mathcal{D}] \equiv P_{0}[\mathcal{D}] r= & \left(q_{1}[\mathcal{D}], \ldots, q_{n}[\mathcal{D}]\right), \quad q_{t}[\mathcal{D}]=\left(\rho_{t}, q_{t 3}[\mathcal{D}]\right), \\
q_{t 3}[\mathcal{D}]= & x_{t 3}-\sum_{j \in \mathcal{D}} k_{j} x_{j 3} M[\mathcal{D}]^{-1}, \quad t \in \mathcal{D}, \\
& q_{t}[\mathcal{D}]=(0,0,0), \quad t \notin \mathcal{D},
\end{aligned}
$$

суть координаты в $R_{0}[\mathcal{D}]$; здесь $M[\mathcal{D}]=\sum_{j \in \mathcal{D}} k_{j}$. Далее, оператор градиента на $R_{03}$ имеет компоненты

$$
\nabla_{t 3}^{\circ}[\mathcal{D}]=k_{t}^{-1} \frac{\partial}{\partial q_{t 3}[\mathcal{D}]}-M[\mathcal{D}]^{-1} \sum_{s \in \mathcal{D}} \frac{\partial}{\partial q_{s 3}[\mathcal{D}]}, \quad t \in \mathcal{D},
$$

и оператор энергии $H_{0}[\mathcal{D}]$ движения кластера $\mathcal{D}$ (относительного движения в направлении третьей оси) может быть записан в виде

$$
H_{0}[\mathcal{D}]=\sum_{t \in \mathcal{D}}\left(T_{t \perp}-k_{t} \nabla_{t 3}^{\circ^{2}}[\mathcal{D}]\right)+\frac{1}{2} \sum_{s, t \in \mathcal{D}, s \neq t} V_{s t}\left(\left|r_{s t}\right|\right) .
$$


1.3. Пусть $Z=\left(C_{1}, \ldots, C_{s}\right)$-произвольное разбиение исходной системы $Z_{1}$ на непересекаюшиеся кластеры $C_{j},|Z|=s$,

$$
V_{Z}=\sum_{j=1}^{s} \sum_{p, t \in C_{j}} V_{p t}, \quad I_{Z}=V-V_{Z}
$$

Определим пространства

$$
\begin{gathered}
R_{0 \perp}(Z)=\sum_{j=1}^{s} \oplus R_{0 \perp}\left[C_{j}\right]=R^{2 n}, \quad R_{03}(Z)=\sum_{j=1}^{s} \oplus R_{03}\left[C_{j}\right], \\
R_{0}(Z)=R_{0 \perp}(Z) \oplus R_{03}(Z), \quad R_{c}(Z)=R_{03} \ominus R_{03}(Z) .
\end{gathered}
$$

Очевидно, $R_{03}(Z)$ и $R_{c}(Z)$ суть соответственно пространства относительного движения $s$-кластерной системы $Z$ и движения ц.м. кластеров $C_{1}, \ldots, C_{s}$ в направлении третьей оси. Ясно, что $R_{0}=R_{0}(Z) \oplus R_{c}(Z)$.

Пусть $P_{03}(Z), P_{0}(Z)$ и $P_{c}(Z)$ - проекторы на $R_{03}(Z), R_{0}(Z)$ и $R_{c}(Z)$. Легко видеть, что

$$
P_{0}(Z) r=\sum_{j=1}^{s} P_{0}\left[C_{i}\right] r, \quad P_{c}(Z) r=\left(\zeta_{1}(Z), \ldots, \zeta_{n}(Z)\right),
$$

где

$$
\zeta_{j}(Z)=\left(0,0, \eta_{j}\right), \quad \eta_{j}=\sum_{i \in C_{t}} k_{i} x_{i 3} M\left[C_{t}\right]^{-1}-M^{-1} \sum_{i=1}^{n} k_{i} x_{i 3} \text { для } j \in C_{t},
$$

$\eta_{j}$ - третья координата вектора, соединяющего центры масс системы $Z_{1}$ и подсистемы $C_{s}$, содержащей $j$. Оператор градиента на $R_{c}(Z)$ имеет компоненты

$$
\nabla_{j 3}^{c}(Z)=M\left[C_{t}\right]^{-1} \nabla_{\eta_{j}}-M^{-1} \sum_{\varrho=1}^{n} \nabla_{\eta_{\varrho}}, \quad j \in C_{t} .
$$

Теперь мы можем записать оператор энергии движения (относительного в направлении третьей оси) составной системы $Z$ в виде

$$
H_{0}(Z)=\sum_{t=1}^{s} H_{0}\left[C_{t}\right]
$$

Легко видеть, что

$$
H(Z) \equiv H_{0}-I_{Z}=H_{0}(Z)+\sum_{t=1}^{n}\left(i \nabla_{t 3}^{c}(Z)\right)^{2}
$$


1.4. Пусть $m$ - тип неприводимого представления группы $S O(2)$ операторами $T_{g}$ : $T_{g} \psi(r)=\psi\left(g^{-1} r\right)\left\{T_{g} \varphi(\rho)=\varphi\left(g^{-1} \rho\right)\right\} g \in S O(2)$ и $P^{(m)}$ - проектор на подпространство функций, которые преобразуются под действием операторов $T_{g}$ по представлению типа $m$. Оператор $P^{(m)}$ коммутирует с гамильтонианами $H_{0}, H_{0}(Z)$. Пусть

$$
\begin{gathered}
H_{0}^{(m)}=P^{(m)} H_{0}, \quad H_{0}^{(m)}(Z)=P^{(m)} H_{0}(Z), \\
\mu^{(m)}=\min _{Z,|Z|=2} \inf H_{0}^{(m)}(Z) .
\end{gathered}
$$

1.5. В пространстве $R^{2 n}$ введем скалярное произведение и норму:

$$
(\rho, \widetilde{\rho})_{2}=\sum_{j=1}^{n} k_{j}\left(\rho_{j}, \widetilde{\rho}_{j}\right)_{R^{2}} M^{-1}, \quad|\rho|_{2}^{2}=(\rho, \rho)_{2},
$$

и определим проекторы (в смысле $\left.(\cdot, \cdot)_{2}\right) P_{0 \rho}$ и $P_{c \rho}$ на подпространства

$$
R_{0 \rho}=\left\{\rho \mid \rho \in R^{2 n}, \sum_{j=1}^{n} k_{j} \rho_{j}=0\right\}, \quad R_{c \rho}=R^{2 n} \ominus R_{0 \rho}
$$

Согласно $[7$, с. 125$]$

$$
\left|P_{0 \rho} \rho\right|_{2}^{2}=\sum_{j=1}^{n} k_{j}\left|\rho_{j}-\rho_{c}\right|^{2}, \quad\left|P_{0 c} \rho\right|_{2}^{2}=M\left|\rho_{c}\right|^{2},
$$

где

$$
\rho_{c}=\sum_{j=1}^{n} k_{j} \rho_{j} M^{-1}
$$

Пусть $C \geq 0, \beta>0$ - произвольные константы,

$$
\begin{gathered}
K(C ; \beta)=\left\{\left.\rho\left|\rho \in R^{2 n},\right| \rho\right|_{2} \geq C,\left|P_{0 \rho} \rho\right|_{2} \leq \beta\left|P_{c \rho} \rho\right|_{2}\right\}, \\
K(0 ; \infty)=R^{2 n}, \quad \mathcal{D}(C ; \beta)=\left\{\varphi(\rho) \mid \varphi(\rho) \in P^{(m)} C_{0}^{2}(K(C ; \beta))\right\} .
\end{gathered}
$$

Замкнем оператор $A$ (см. п. 1.1) с $\mathcal{D}(C ; \beta)$; обозначим полученный оператор через $A^{(m)}(C ; \beta)$ и положим

$$
\begin{gathered}
A_{1}^{(m)}(C ; \beta)=\left(A^{(m)}(C, \beta)+I\right)^{-1}, \quad A^{(m)}(\beta)=A^{(m)}(0, \beta), \quad A_{1}^{(m)}(\beta)=A_{1}^{(m)}(0, \beta), \\
A^{(m)}=A^{(m)}(+\infty), \quad A_{1}^{(m)}=A_{1}^{(m)}(+\infty) .
\end{gathered}
$$

Пусть $S_{d}$ - класс всех самосопряженных операторов, имеюших чисто дискретный спектр с единственной предельной точкой $+\infty$. Наконец, обозначим существенный спектр произвольного оператора $h$ через $\sum_{\mathrm{ess}}(h)$. 


\section{2. РЕЗУЛЬТАТЫ}

2.1. В настояшем разделе мы формулируем и обсуждаем полученные результаты.

Теорема 2.1. Пусть для некоторого $\beta>0$ оператор $A_{1}^{(m)}(\beta)$ компактен. Тогда

$$
\sum_{\text {ess }}\left(H_{0}^{(m)}\right)=\left[\mu^{(m)},+\infty\right)
$$

ЗАмЕчАниЕ. Компактность оператора $A_{1}^{(m)}(\beta)$ эквивалентна включению $A^{(m)}(\beta) \in S_{d}$.

2.2. Обсудим физический смысл условия теоремы 2.1 и его отличие от условий соответствующих теорем в работах $[1,2]$. Мы знаем, что для каждой точки $\lambda$ из $\sum_{\text {ess }}\left(H_{0}^{(m)}\right)$ сушествует такая последовательность (последовательность Вейля) $\psi_{t}(r) \in$ $P^{(m)} C_{0}^{2}\left(R_{0}\right),\left\|\psi_{t}\right\|=1$, что

$$
\psi_{t}(r) \rightarrow 0 \quad \text { в } \mathcal{L}_{2}\left(R_{0}\right), \quad \lambda=\lim _{t \rightarrow \infty}\left(H_{0} \psi_{t}, \psi_{t}\right) .
$$

Легко видеть, что для этой последовательности

$$
\lim _{t \rightarrow \infty} \int_{\Omega}\left|\psi_{t}\right|^{2} d \Omega=0
$$

для любой компактной области $\Omega \subset R_{0}$. Это значит, что $\psi_{t}(r)$ является такой последовательностью состояний нашей системы, которая описывает уход системы из любой компактной области конфигурационного пространства $R_{0}$. Следовательно, $\psi_{t}(r)$ может описывать один из двух случаев:

а) какое-либо распадение $Z_{1}$ на два или более кластеров, возможно, с одновременным движением ц.м. к бесконечности;

б) движение ц.м. системы к бесконечности без какого-либо распадения.

Понятно, что в случае "а" естественно ожидать, что

$$
\lambda=\lim _{t \rightarrow \infty}\left(H_{0}^{(m)} \psi_{t}, \psi_{t}\right) \geq \mu^{(m)},
$$

т.к. $\lambda$ есть предел значений энергии системы для состояния $\psi_{t}(r)$, а $\mu^{(m)}$ - минимальное значение энергии по всем распадениям системы при фиксированном значении полного углового момента ${ }^{2)}$.

В случае "б" неравенство (2.3) может не вьполняться, и это значит, что утверждение (2.1) может быть неверным. Поэтому главная задача локализации существенного спектра для систем с магнитным полем состоит в нахождении условий, при которых любая последовательность Вейля не может отвечать случаю "б”, т.е. не может описывать движение ц.м. системы $Z_{1}$ к бесконечности без какого-либо распадения.

\footnotetext{
${ }^{2)}$ Доказательство неравенства (2.3) для $\forall \lambda \in \sum_{\mathrm{ess}}\left(H_{0}^{(m)}\right)$ является “тяжелой” частью всех геометрических доказательств утверждений о локализации существенного спектра многочастичных систем, т.е. утверждений типа ХВЖК-теоремы.
} 
2.3. Ясно, что $\psi_{t}(r)$ не может соответствовать движению ц.м. к бесконечности в направлении третьей оси, т.к. в $R_{0}$ третья координата ц.м. фиксирована.

Возможность движения ц.м. к бесконечностив плоскости $x_{1}, x_{2}$ зависит от характера взаимодействия магнитного поля с частицами. Это взаимодействие определяет спектральные свойства операторов $A^{(m)}(\beta), A_{1}^{(m)}(\beta), 0<\beta \leq+\infty$, а эти свойства, в свою очередь, могут определять главные черты поведения системы в магнитном поле. Так, например, условие компактности оператора $A_{1}^{(m)}$ (которое было введено в [2] для доказательства теоремы о существенном спектре, но фактически использовалось ранее в [1]) запрещает движение ц.м. системы к бесконечности в плоскости $x_{1}, x_{2}$, поскольку такое движение приводит к бесконечному возрастанию энергии. В самом деле, если $\psi_{t}(r)$ описывает такое движение, то

$$
\delta=\lim _{k \rightarrow \infty} \sup _{t}\left\|\psi_{t}\right\|_{\left|\rho_{c}\right| \geq k}>0
$$

но в силу компактности оператора $A_{1}^{(m)}$ оператор $A^{(m)} \in S_{d}$, и, следовательно, если $\delta>0$, то мы получаем

$$
\lim \left(A^{(m)} \psi_{t}, \psi_{t}\right)=+\infty,
$$

что противоречит неравенству $\lambda<+\infty$.

2.4. К несчастью, компактность оператора $A_{1}^{(m)}$ запрешает последовательности Вейля отвечать любому движению ц.м. к бесконечности в плоскости $x_{1}, x_{2}$, включая такое движение, которое сопровождается распадением системы и не противоречит (2.3). Условие компактности оператора $A_{1}^{(m)}(\beta)$ в теореме 2.1 не имеет этого недостатка, т.к. оно запрещает движение ц.м. к бесконечности только в конусе $K(0, \beta)$. Вне данного конуса такое движение возможно, но оно всегда сопровождается распадением системы, т.к. вне этого конуса в силу (1.5)

$$
\sum_{j=1}^{n} k_{j}\left|\rho_{j}-\rho_{c}\right|^{2} \geq \beta^{2} M\left|\rho_{c}\right|^{2}
$$

и если $\left|\rho_{c}\right| \rightarrow \infty$, то $\left|\rho_{j}-\rho_{c}\right| \rightarrow \infty$ для какого-либо $j$, т.е. система распадается.

2.5. Достаточные условия компактности оператора $A_{1}^{(m)}(\beta)$ даны в следующей теореме.

ТЕОРема 2.2. Положим

$$
Q=\sum_{j=1}^{n} e_{j}, \quad Q_{0}=\sum_{j=1}^{n}\left|e_{j}\right|
$$

Пусть $Q \neq 0$ и функиия $B(t)$, определяющая вид магнитного вектор-потенииала (см. п. 1.3), удовлетворяет соотношению

$$
\lim _{t \rightarrow \infty}|B(t) t|=\infty
$$


и пусть выполняется по крайней мере одно из следующих условий:

или для некоторых $q>0, C_{0}>0, \delta_{0}>0$

$$
\left|B(t) B(s)^{-1}-1\right| \leq q<|Q| Q_{0}^{-1}
$$

$\kappa о г д а\left|t s^{-1}-1\right| \leq \delta_{0}, \quad t, s \geq C_{0}$,

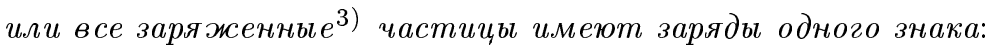

$$
e_{i} e_{j} \geq 0 \quad \forall i, j
$$

Тогда существует такое $\bar{\beta}$, что при $\beta<\bar{\beta}$ оператор $A_{1}^{(m)}(\beta)$ компактен.

Теоремы 2.1, 2.2 могут быть применены к широкому классу не нейтральных квантовых систем и магнитных вектор-потенциалов с невозрастающими функциями $B(t)$. Например, в силу теоремы 2.2 оператор $A_{1}^{(m)}(\beta)$ компактен для малых $\beta$ (и, следовательно, утверждение (2.1) выполняется), когда

$$
B(t)=a t^{\alpha}(1+\gamma \sin \omega t)
$$

где $\alpha, \gamma$ - такие вещественные константы, что $-1<\alpha \leq 0,|\gamma|<|Q| \cdot\left(2 Q_{0}+|Q|\right)^{-1}$, $a, \omega$ - любые положительные числа.

Условия (2.4), (2.5) теоремы 2.2 можно заменить ограничениями на магнитное поле $F\left(\left|\rho_{1}\right|\right)=\left(0,0, F_{3}\left(\left|\rho_{1}\right|\right)\right)$ (для которого $B\left(\left|\rho_{1}\right|\right)\left(-x_{12}, x_{11}, 0\right)$ - соответствующий магнитньй вектор-потенциал). В общем случае мы этого не делаем, но полезно заметить, что условия (2.4), (2.5) выполняются, например, если

$$
F_{3}(t)=a t^{\alpha}(1+\gamma \sin \omega t)
$$

где $a, \gamma, \omega$ - произвольные действительные числа, $-1<\alpha \leq 0$.

Заметим, что случай $\gamma=\alpha=0$ был изучен ранее в [6] для систем без нейтральных частиц. По сравнению с $[1,2]$ наши теоремы позволяют отдельным частицам иметь различные знаки зарядов или нулевой заряд (что было запрешенно в $[1,2]$ ).

Доказательства теорем 2.1, 2.2 даны соответственно в разделах 3,4 .

Так как область $K(0, \beta) \backslash K(C, \beta)$ ограничена, то операторы $A_{1}^{(m)}(C ; \beta)$ и $A_{1}^{(m)}(\beta)$ компактны и некомпактны одновременно для любого $C$. Поэтому далее вместо оператоpa $A_{1}^{(m)}(\beta)$ мы рассматриваем оператор $A_{1}^{(m)}(C ; \beta)$ для больших $C>0$.

\footnotetext{
${ }^{3)}$ Система может содержать нейтральные частицы, но все частицы не могут быть нейтральными, ибо $Q \neq 0$.
} 


\section{3. ДОКАЗАТЕЛЬСТВО ТЕОРЕМЫ 2.1}

3.1. Доказательство теоремы 2.1 состоит в проверке включений

$$
\left[\mu^{(m)},+\infty\right) \subseteq \sum_{\text {ess }}\left(H_{0}^{(m)}\right)
$$

и

$$
\sum_{\text {ess }}\left(H_{0}^{(m)}\right) \subseteq\left[\mu^{(m)},+\infty\right) .
$$

Первое включение доказывается тождественно, так же как в [1] (п. 2.7, 2.8). Чтобы доказать включение (3.1), достаточно установить, что для любой последовательности

$$
\left\{g_{k}(r)\right\}, \quad g_{k}(r) \in P^{(m)} C_{0}^{2}\left(R_{0}\right), \quad\left\|g_{k}\right\|=1, \quad g_{k}(r) \rightarrow 0 \text { в } \mathcal{L}_{2}\left(R_{0}\right),
$$

выполняется

$$
\underline{\varliminf}\left(H_{0}^{(m)} g_{k}, g_{k}\right) \geq \mu^{(m)} .
$$

Доказательство (3.2) состоит из двух частей. В первой части мы разбиваем пространство $R_{0}$ на области, соответствующие разбиениям $Z_{s}=\left(C_{1}, \ldots, C_{s}\right)$ системы $Z_{1}=(1,2, \ldots, n)$, возникаюшим в результате возрастания расстояний между кластерами $C_{j}$ только в направлении оси $x_{3}$, т.е. мы делаем разбиения только в пространстве $R_{03}$. Заметим, что ни одна из построенных областей не отвечает движению ц.м. к бесконечности в направлении оси $x_{3}$, т.к. в $R_{03}$ положение ц.м. фиксировано. Данное разбиение (3.5) осушествляется функциями (3.4), которые реализуют разбиение единицы. Далее, мы оцениваем квадратичную форму оператора $H_{0}^{(m)}$ по полученным областям, за исключениям цилиндра

$$
\Omega=\left\{\left.X_{3}\left|X_{3} \in R_{03},\right| X_{3}\right|_{1} \leq b(1)\right\} \otimes R^{2 n},
$$

где $b(1)$ - некоторая константа.

Доказательство этой части теоремы не отличается от [1] (п. 2.4, 2.5), и мы проводим его очень кратко.

Во второй части доказательства мы оцениваем квадратичную форму оператора $H_{0}^{(m)}$ в $\Omega$. Подход [1] не может быть применен для этой оценки, т.к. оператор $A_{1}^{(m)}$ не компактен в $R^{2 n}$ для систем, которые содержат частицы с зарядами разных знаков или нейтральные частишы.

Чтобы оценить $\left(H_{0}^{(m)} g_{k}, g_{k}\right)_{\Omega}$, мы делаем специальное разбиение области $\Omega$. Главные особенности этого разбиения состоят в следуюшем:

а) оно порождено разбиением пространства $R^{2 n}$;

б) во всех построенных областях (исключая $\Omega_{-1}$ ) возможно движение ц.м. системы к бесконечности в плоскости $x_{1}, x_{2}$;

в) только в одной области $\Omega_{0}$ такое движение логически возможно без распадения системы.

Мы осуществляем это специальное разбиение с помощью функций (3.8) и далее оцениваем квадратичную форму оператора $H_{0}^{(m)}$ по полученным областям. Заметим, что компактность оператора $A_{1}^{(m)}(\beta)$ используется для оценки только в области $\Omega_{0}$ : мы доказываем, что функции $g_{k}$ описьвают уход рассматриваемой системы из области $\Omega_{0}$. 
3.2. Итак, сначала мы следуем [1] . Пусть $Z_{s}=\left(C_{1}, \ldots, C_{s}\right), P_{03}\left(Z_{s}\right)$ и $P_{c}\left(Z_{s}\right)$ те же, что в п. 1.3 при

$$
\begin{aligned}
& Z=Z_{s}, \quad X_{3}=\left(x_{13}, x_{23}, \ldots, x_{n 3}\right), \quad\left|X_{3}\right|_{1}^{2}=\sum_{i=1}^{n} x_{i 3}^{2} k_{i}, \\
& \tau_{1}=\left|X_{3}\right|_{1}, \quad \tau_{s}=\tau\left(Z_{s}\right)=\left|P_{03}\left(Z_{s}\right) X_{s}\right| \cdot\left|P_{c}\left(Z_{s}\right) X_{3}\right|^{-1} .
\end{aligned}
$$

Мы выбираем большие числа $a(1)<b(1)$ и малые числа $a(s)<b(s), s \geq 2$, так же, как в п. 2.4 работы [1] , и определим такие вещественные кусочно-непрерывно дифференцируемые функции $u_{s}(t), v_{s}(t)$, что $0 \leq u_{s}(t), v_{s}(t) \leq 1, u_{s}(t)=1$ для $t<a(s), u_{s}(t)=0$ для $t \geq b(s)$,

$$
u_{s}^{2}(t)+v_{s}^{2}(t)=1 .
$$

Пусть $u_{Z_{s}}=u_{s}\left(\tau\left(Z_{s}\right)\right), v_{Z_{s}}=v_{s}\left(\tau\left(Z_{s}\right)\right), \quad \psi(r)=g_{k}(r)$

$$
\begin{gathered}
\hat{\psi}_{0} \equiv \psi, \quad \hat{\psi}_{i}=\hat{\psi}_{i-1}\left(1-\sum_{Z_{i}} u_{Z_{i}}^{2}\right)^{1 / 2}, \quad \psi_{i-1, Z_{i}}=\hat{\psi}_{i-1} u_{Z_{i}}, \quad i=1, \ldots, n, \\
\psi_{i}=\sum_{Z_{i}} \psi_{i-1, Z_{i}}, \quad i=1,2, \ldots, n-1 .
\end{gathered}
$$

Тогда согласно соотношениям (2.5), (2.6) из [1] мы получим для $\forall \varepsilon>0$ и большого $a(1)=a(1, \varepsilon)$

$$
\begin{gathered}
\left(H_{0} g_{k}, g_{k}\right) \geq \sum_{j=0}^{n-1} \sum_{Z_{j+1}}\left(H\left(Z_{j+1}\right) \psi_{j, Z_{j+1}}, \psi_{j, Z_{j+1}}\right)-2 \varepsilon\left\|g_{k}\right\|^{2}, \\
\left(H\left(Z_{j+1}\right) \psi_{j, Z_{j+1}}, \psi_{j, Z_{j+1}}\right) \geq \mu^{(m)}\left\|\psi_{j, Z_{j+1}}\right\|^{2}, \quad j \neq 0 .
\end{gathered}
$$

3.3. Оценим теперь величину $\left(H\left(Z_{1}\right) \psi_{0, Z_{1}}, \psi_{0, Z_{1}}\right)$. Мы учтем, что $\operatorname{supp} \psi_{0, Z_{1}} \subseteq \Omega$, где

$$
\Omega=\left\{\left.r\left|r=\left(r_{1}, \ldots, r_{n}\right), r \in R_{0},\right| X_{3}\right|_{1} \leq b(1)\right\},
$$

и разобьем $\Omega$ с помошью разложения пространства $R^{2 n}$ на области, отвечаюшие всем возможным вариантам распадения системы $Z_{1}$ в плоскости $x_{1}, x_{2}$. При этом в отличие от ситуации п. 3.2 наше разбиение содержит области, которые соответствуют возможному движению ц.м. системы $Z_{1}$ к бесконечности в плоскости $x_{1}, x_{2}$.

Для произвольного разбиения $Z_{s}=\left(C_{1}, \ldots, C_{s}\right)$ положим

$$
\begin{gathered}
R_{0 \rho}\left(Z_{s}\right)=\left\{\rho \mid \rho \in R_{\rho}, \sum_{j \in C_{i}} k_{j} \rho_{j}=0 i=1, \ldots, s\right\}, \\
R_{c \rho}\left(Z_{1}\right)=R_{\rho} \ominus R_{0 \rho}\left(Z_{1}\right), \quad R_{c \rho}\left(Z_{s}\right)=R_{0 \rho}\left(Z_{1}\right) \ominus R_{0 \rho}\left(Z_{s}\right), \quad s \geq 2 .
\end{gathered}
$$

Пусть $P_{\gamma \rho}\left(Z_{s}\right)$ - проектор в смысле $(\cdot, \cdot)_{2}$ (см. п. 1.5) на подпространство $R_{\gamma \rho}\left(Z_{s}\right)$, $\gamma=0, c, \chi_{0}=\chi\left(Z_{0}\right)=|\rho|_{2}{ }^{4)}, \chi_{s}=\chi\left(Z_{s}\right)=\left|P_{0 \rho}\left(Z_{s}\right) \rho\right|_{2} \cdot\left|P_{c \rho}\left(Z_{s}\right) \rho\right|_{2}^{-1}, s \geq 1$.

\footnotetext{
${ }^{4)}$ Обозначение $Z_{0}$ введено только для единства обозначений.
} 
Определим функции $\widetilde{u}_{s}(t), \widetilde{v}_{s}(t)$ аналогично $u_{s}(t), v_{s}(t)$, но сейчас $\widetilde{u}_{s}(t)=1$ для $t \leq a(s+1), \widetilde{u}_{s}(t)=0$ для $t \geq b(s+1)$. Далее, положим

$$
\begin{gathered}
\widetilde{u}_{Z_{s}}=\widetilde{u}_{s}\left(\chi\left(Z_{s}\right)\right), \quad \tilde{v}_{Z_{s}}=\widetilde{v}_{s}\left(\chi\left(Z_{s}\right)\right), \quad s=0,1, \ldots, n, \\
\varphi=\psi_{0, Z_{1}}, \quad \hat{\varphi}_{-1}=\varphi, \quad \hat{\varphi}_{i}=\hat{\varphi}_{i-1}\left(1-\sum_{Z_{i}} \widetilde{u}_{Z_{i}}^{2}\right)^{1 / 2}, \\
\varphi_{i-1, Z_{i}}=\hat{\varphi}_{i-1} \widetilde{u}_{Z_{i}}, \quad i=0,1, \ldots, n .
\end{gathered}
$$

Заметим, что функция $\widetilde{u}_{Z_{0}}=\widetilde{u}_{0}\left(|\rho|_{2}\right)$ описывает ситуацию, когда все частицы находятся в ограниченной области пространства $R^{2 n}$ (эта функция “работает” так же, как функция $u_{Z_{1}}\left(\tau_{1}\right)$ в $\left.R_{03}\right)$, а функция $\widetilde{u}_{Z_{1}}$ соответствует возможному движению ц.м. к бесконечности в конусе $K(0 ; b(2))$.

В силу выбора чисел $a(s), b(s)$ справедливо соотношение

$$
\operatorname{supp} \varphi_{i, Z_{i+1}} \cap \operatorname{supp} \varphi_{i, Z_{i+1}^{\prime}}=\varnothing, \text { если } Z_{i+1} \neq Z_{i+1}^{\prime}
$$

(см. следствие 1 леммы 3.5 из [8]).

3.4. Для большого $b(1)$ мы можем получить соотношение, аналогичное (2.3) из работы [1]:

$$
\left(H_{0} \varphi, \varphi\right) \geq \sum_{i=-1}^{n-1} \sum_{Z_{i+1}}\left(H_{0} \varphi_{i, Z_{i+1}}, \varphi_{i, Z_{i+1}}\right)-C\left\|\hat{\varphi}_{0}|\rho|^{-1}\right\|^{2}-\varepsilon\left\|\varphi-1, Z_{0}\left(1+|\rho|_{2}\right)^{-1}\right\|^{2}
$$

где $\varepsilon-$ произвольно малое число, $C$ и $b(1)$ зависят от $\varepsilon$. Если $r \in \operatorname{supp} \varphi_{i, Z_{i+1}}$, то для $j, t$ из различных кластеров разбиения $Z_{i+1}, i \geq 1$,

$$
\left|\rho_{j}-\rho_{t}\right| \geq a(1) \cdot \mathrm{const}
$$

(см. лемму 3.7 и соотношения (2.18) из [8]).

Следовательно, при $i \neq-1,0$, произвольном $\varepsilon_{1}>0$ и большом $a(1)$

$$
\left(H_{0} \varphi_{i, Z_{i+1}}, \varphi_{i, Z_{i+1}}\right) \geq\left(H\left(Z_{i+1}\right) \varphi_{i, Z_{i+1}}, \varphi_{i, Z_{i+1}}\right)-\varepsilon_{1}\left\|\varphi_{i, Z_{i+1}}\right\|^{2}
$$

и аналогично (3.7)

$$
\left(H\left(Z_{i+1}\right) \varphi_{i, Z_{i+1}}, \varphi_{i, Z_{i+1}}\right) \geq \mu^{(m)}\left\|\varphi_{i, Z_{i+1}}\right\|^{2}
$$


3.5. Оценим теперь величину $\left(H_{0} \varphi_{i, Z_{i+1}}, \varphi_{i, Z_{i+1}}\right)$ при $i=-1,0$. По построению

$$
\varphi_{i, Z_{i+1}}(r)=g_{k}(r) \omega_{i}(r),
$$

где $\omega_{i}(r) \in C^{2}$

$$
\begin{gathered}
\sup _{r, j, k, p, l}\left\{\left|\omega_{i}(r)\right|+\left|\frac{\partial \omega_{i}}{\partial x_{j k}}\right|+\left|\frac{\partial^{2} \omega_{i}}{\partial x_{j k} \partial x_{p l}}\right|\right\}<+\infty, \quad i=-1,0, \\
\Omega_{-1} \equiv \operatorname{supp}\left\{\omega_{-1}(r)\right\}=\left\{\left.r\left|r \in R_{0},\right| X_{3}\right|_{1} \leq b(1),|\rho|_{2} \leq b(1)\right\}, \\
\Omega_{0} \equiv \operatorname{supp}\left\{\omega_{0}(r)\right\}=\left\{\left.r\left|r \in R_{0},\right| X_{3}\right|_{1} \leq b(1), \rho \in K(b(1) ; b(2))\right\}, \\
\omega_{i}(r)=0, \quad r \in \partial \Omega_{i} .
\end{gathered}
$$

Обозначим через $H_{0 i}$ замыкание оператора $H_{0}$ в $\mathcal{L}_{2}\left(\Omega_{i}\right)$ области $P^{(m)} C_{0}^{2}\left(\Omega_{i}\right)$.

Покажем, что операторы $H_{0 i}$ принадлежат $S_{d}$ (определение $S_{d}$ см. в п. 1.5). Для $H_{0,-1}$ это очевидно, т.к. область $\Omega_{-1}$ ограничена. Для оператора $H_{0,0}$ имеем аналогично лемме 2.1 из [1]

$$
H_{0,0}^{(m)} \geq h \equiv\left(\delta_{1} A(b(2))-\delta_{2} \nabla_{03}^{2}-\delta_{3}\right) P^{(m)},
$$

где $\delta_{i}$ - некоторые положительные константы. Так как в области $\Omega_{0}$ выполняется $\left|X_{3}\right|_{1} \leq b(1)$ и $\rho \in K(b(1) ; b(2))$, то при $b(2)<\beta$ с помощью разделения переменных заключаем, что $h \in S_{d}$. Следовательно, и $H_{0,0}^{(m)} \in S_{d}$.

Далее, в силу п. 3.1 и неравенства (3.6) последовательность $g_{k}(r) \omega_{i}(r)$ обладает свойствами, необходимыми для применения леммы 3.1, п. 3.7 (см. ниже). В силу этой леммы при $i=-1$ и $i=0$

$$
\begin{gathered}
\frac{\lim }{k \rightarrow \infty}\left(H_{0} \varphi_{i, Z_{i+1}}, \varphi_{i, Z_{i+1}}\right)=\lim _{k \rightarrow \infty}\left(H_{0} g_{k} \omega_{i}, g_{k} \omega_{i}\right) \geq 0 \\
\lim _{k \rightarrow \infty}\left\|\varphi_{i, Z_{i+1}}\right\|=\lim _{k \rightarrow \infty}\left\|g_{k} \omega_{i}\right\|=0
\end{gathered}
$$

3.6. По построению

$$
\left\|\psi_{0, Z_{1}}\right\|^{2}=\|\varphi\|^{2}=\sum_{i=-1}^{n-1} \sum_{Z_{i+1}}\left\|\varphi_{i, Z_{i+1}}\right\|^{2} .
$$

В силу (3.9)-(3.11) имеем

$$
\left(H_{0} \psi_{0, Z_{1}}, \psi_{0, Z_{1}}\right) \geq\left(\mu^{(m)}-\varepsilon_{2}\right)\left\|\psi_{0, Z_{1}}\right\|^{2}-\mu^{(m)}\left\|g_{k} \omega_{-1}\right\|^{2}-\mu^{(m)}\left\|g_{k} \omega_{0}\right\|^{2}+\gamma_{k},
$$

где $\gamma_{k}=\left(H_{0} g_{k} \omega_{-1}, g_{k} \omega_{-1}\right)+\left(H_{0} g_{k} \omega_{0}, g_{k} \omega_{0}\right), \varepsilon_{2}$ мало, если $a(1)$ велико.

В силу (3.5)-(3.7), (3.15) и так как

$$
\operatorname{supp} \psi_{j, Z_{j+1}} \cap \operatorname{supp} \psi_{j, Z_{j+1}^{\prime}}=\varnothing, \quad \text { если } Z_{j+1} \neq Z_{j+1}^{\prime}
$$

(см. следствие леммы 3.5 из [8]), мы получаем, что

$$
\left(H_{0} g_{k}, g_{k}\right) \geq\left(\mu^{(m)}-\varepsilon_{3}\right)\left\|g_{k}\right\|^{2}-\mu^{(m)}\left(\left\|g_{k} \omega_{-1}\right\|^{2}+\left\|g_{k} \omega_{0}\right\|^{2}\right)+\gamma_{k},
$$

где $\varepsilon_{3}$ мало.

В силу (3.12), (3.13)

$$
\lim _{k \rightarrow \infty} \gamma_{k} \geq 0, \quad \lim _{k \rightarrow \infty}\left\|g_{k} \omega_{i}\right\|=0, \quad i=-1,0
$$

Поэтому неравенство (3.2) следует из (3.16). 
3.7. Докажем утверждение, использованное в п. 3.6.

Лемма 3.1. Пусть $T$ - самосопряженный оператор в $\mathcal{L}_{2}\left(R_{0}\right)$ с областью $D_{T} u$ $T \in S_{d}$. Тогда для любой последовательности $f_{k}(r) \in D_{T},\left\|f_{k}\right\| \leq 1, f_{k} \rightarrow 0$ в $\mathcal{L}_{2}\left(R_{0}\right)$, для которой

$$
\varlimsup
$$

вьполняются соотношения

$$
\underline{\lim }\left(T f_{k}, f_{k}\right) \geq 0, \quad \lim _{k \rightarrow \infty}\left\|f_{k}\right\|=0
$$

ДокАЗАТЕЛЬСТво тривиально. Действительно, пусть $g_{s}(r)$ - полная система ортонормированных собственных функций оператора $T$ и $\lambda_{1} \leq \lambda_{2} \leq \cdots \leq \lambda_{s} \leq \ldots$ соответствующие собственные значения. Тогда

$$
\begin{gathered}
f_{k}(r)=\sum_{s=1}^{\infty}\left(f_{k}, g_{s}\right) g_{s}(r), \quad\left\|f_{k}\right\|^{2}=\sum_{s=1}^{\infty}\left|\left(f_{k}, g_{s}\right)\right|^{2}, \\
\left(T f_{k}, f_{k}\right)=\sum_{s=1}^{\infty} \lambda_{s}\left|\left(f_{k}, g_{s}\right)\right|^{2}
\end{gathered}
$$

По условию леммы $\left(f_{k}, g_{s}\right) \rightarrow 0$, если $k \rightarrow \infty$, поэтому для любого фиксированного $N$

$$
\underline{\lim }\left(T f_{k}, f_{k}\right) \geq \lambda_{N} \lim \sum_{s=N}^{\infty}\left|\left(f_{k}, g_{s}\right)\right|^{2}=\lambda_{N} \lim _{k \rightarrow \infty}\left\|f_{k}\right\|^{2}
$$

Т.к. $\lambda_{N} \rightarrow \infty$ при $N \rightarrow \infty$, то $\lim _{k \rightarrow \infty}\left\|f_{k}\right\|=0$, и лемма доказана.

\section{4. ДОКАЗАТЕЛЬСТВО ТЕОРЕМЫ 2.2}

4.1. Для доказательства теоремы 2.2 достаточно показать, что

$$
\lim \left(A \varphi_{t}, \varphi_{t}\right)=+\infty
$$

для произвольной последовательности $\varphi_{t}(\rho) \in \mathcal{D}(C ; \beta),\|\varphi\|_{R_{\rho}}=1$, для которой

$$
\lim \left\|\varphi_{t}(\rho)\right\|_{\Omega}=0
$$

для каждой ограниченной области $\Omega \subset R_{\rho}$.

Т.к. $\varphi_{t}(\rho)=P^{(m)} \varphi_{t}(\rho)$, то можно разложить функцию $\varphi_{t}(\rho)$ по собственным функциям оператора полного углового момента, которые отвечают собственному значению $m$. Пусть $\varphi(\rho) \equiv \varphi_{t}(\rho)$. Имеем

$$
\varphi(\rho)=\sum(m) \varphi_{m_{1}, \ldots, m_{n}}(\rho)
$$


где $\sum(m)$ есть сумма по всем целочисленным $m_{1}, \ldots, m_{n}, m_{1}+\cdots+m_{n}=m$,

$$
\begin{gathered}
\varphi_{m_{1}, \ldots, m_{n}}(\rho)=e^{-i\left(m_{1} \gamma_{1}+\cdots+m_{n} \gamma_{n}\right)} f_{m_{1}, \ldots, m_{n}}(\varrho) \\
f_{m_{1}, \ldots, m_{n}}(\varrho)=(2 \pi)^{-n} \int_{0}^{2 \pi} \cdots \int_{0}^{2 \pi} \varphi(\rho) e^{i\left(m_{1} \gamma_{1}+\cdots+m_{n} \gamma_{n}\right)} d \gamma_{1} \ldots d \gamma_{n}
\end{gathered}
$$

$\varrho=\left(\varrho_{1}, \ldots, \varrho_{n}\right), \varrho_{j}, \gamma_{j}$ суть полярные координаты в плоскости $x_{1}, x_{2}$ :

$$
x_{j 1}=\varrho_{j} \cos \gamma_{j}, \quad x_{j 2}=\varrho_{j} \sin \gamma_{j} .
$$

Очевидно, что

$$
(A \varphi, \varphi)=\sum(m)\left(A \varphi_{m_{1}, \ldots, m_{n}}, \varphi_{m_{1}, \ldots, m_{n}}\right)
$$

$$
\left(A \varphi_{m_{1}, \ldots, m_{n}}, \varphi_{m_{1}, \ldots, m_{n}}\right) \geq(2 \pi)^{n}\left(F_{m_{1}, \ldots, m_{n}}(\varrho) f_{m_{1}, \ldots, m_{n}}, f_{m_{1}, \ldots, m_{n}}\right)_{R^{n}},
$$

где

$$
F_{m_{1}, \ldots, m_{n}}(\varrho)=\sum_{j=1}^{n} k_{j}^{-1}\left(m_{j}^{2} \varrho_{j}^{-2}+2 m_{j} e_{j} B_{j}+e_{j}^{2} B_{j}^{2} \varrho_{j}^{2}\right), \quad B_{j}=B\left(\varrho_{j}\right), \quad R^{n}=\{\varrho\} .
$$

Рассматривая, далее, $m_{1}, \ldots, m_{n}$ как непрерывные параметры, минимизируем $F_{m_{1}, \ldots, m_{n}}(\varrho)$ для фиксированного значения $\varrho$ по $m_{1}, \ldots, m_{n}$ при условии $m_{1}+m_{2}+$ $\cdots+m_{n}=m$. Тогда мы получим следуюшее неравенство:

$$
F_{m_{1}, \ldots, m_{n}}(\varrho) \geq F_{m}(\varrho) \equiv\left(m+\sum_{j=1}^{n} e_{j} B_{j} \varrho_{j}^{2}\right)^{2}\left(\sum_{j=1}^{n} k_{j} \varrho_{j}^{2}\right)^{-1} .
$$

4.2. Оценим теперь $F_{m}(\varrho)$ при $\varrho \in \operatorname{supp} f_{m_{1}, \ldots, m_{n}}(\varrho)$. С этой целью опишем множество $\operatorname{supp} f_{m_{1}, \ldots, m_{n}}(\varrho)$. В силу (1.5)

$$
|\rho|_{2}^{2}=\left|P_{0 \rho} \rho\right|_{2}^{2}+\left|P_{c \rho}\right|_{2}^{2} .
$$

Если $\rho \in K(C ; \beta)$, то в силу $(1.5)$

$$
\left.\left|\varrho_{i}\right| \rho_{c}\right|^{-1}-\left.1\left|\leq \beta_{1}, \quad\right| \rho_{c}|\geq| \rho\right|_{2}\left(1+\beta_{1}^{2}\right)^{-1 / 2} \geq N_{1},
$$

где $\beta_{1}=\beta M^{1 / 2} k_{0}^{-1 / 2}, N_{1}=C\left(1+\beta_{1}^{2}\right)^{-1 / 2} M^{-1 / 2}, k_{0}=\min _{j} k_{j}$.

Вследствие (4.11) для $\rho \in K(C ; \beta)$ имеем

$$
\left|\varrho_{i} \varrho_{j}^{-1}-1\right| \leq \beta_{2}, \quad \varrho_{j} \geq\left(1-\beta_{1}\right)\left|\rho_{c}\right| \geq N_{2}, \quad i, j=1,2, \ldots, n,
$$

где $\beta_{2}=2 \beta_{1}\left(1-\beta_{1}\right)^{-1}, N_{2}=\left(1-\beta_{1}\right) N_{1}$.

Поскольку $\operatorname{supp} \varphi(\rho) \subset K(C, \beta)$, то ясно, что $\varphi\left(\rho_{1}, \ldots, \rho_{n}\right) \equiv 0$ для всех углов $\gamma_{1}, \ldots, \gamma_{n}$, если для каких-либо значений $i, j, t$

$$
\left|\varrho_{i} \varrho_{j}^{-1}-1\right|>\beta_{2} \text { или } \varrho_{t}<N_{2} \text {. }
$$

В силу (4.5) $f_{m_{1}, \ldots, m_{n}}(\varrho) \equiv 0$, если выполняется (4.13), и, значит, при $\varrho \in$ $\operatorname{supp} f_{m_{1}, \ldots, m_{n}}(\varrho)$ верно неравенство (4.12). Выберем, далее, $\beta$ столь малым и $C$ столь большим, что $\beta_{2}<\delta_{0}$ и $N_{2}>C_{0}$.

2 Теоретическая и математическая физика, т. 107, № 3, 1996 г. 
4.3. Рассмотрим случай, когда в системе имеются частицы с зарядами разных знаков. Для $\forall s$ и $\varrho \in \operatorname{supp} f_{m_{1}, \ldots, m_{n}}(\varrho)$ в силу (4.12) имеем

$$
\varrho_{j} \varrho_{s}^{-1}=1+\beta_{j s}, \quad B_{j} B_{s}^{-1}=1+q_{j s},
$$

где $\beta_{j s}$ и $q_{j s}$ - некоторые функции, $\left|\beta_{j s}\right|<\beta_{2} \leq 1,\left|q_{j s}\right| \leq q$.

Используя (4.14) и (2.5), мы получаем

$$
\begin{aligned}
\left|\sum_{j=1}^{n} e_{j} B_{j} \varrho_{j}^{2}+m\right| & =\left|B_{s}\right| \varrho_{s}^{2}\left|\sum^{\prime} e_{j}\left(1+q_{j s}\right)\left(1+\beta_{j s}\right)^{2}+m \varrho_{s}^{-2} B_{s}^{-1}\right| \geq \\
& \geq\left|B_{s}\right| \varrho_{s}^{2}\left[|Q|-Q_{0}\left(q+3 \beta_{2}+3 \beta_{2} q+m \varrho_{s}^{-2}\left|B_{s}\right|^{-1} Q_{0}^{-1}\right)\right],
\end{aligned}
$$

где $\sum^{\prime}$ есть сумма по всем $j$, для которых $e_{j} \neq 0$, и $s$ есть одно из таких $j$.

В силу $(2.4),(2.5)$ и (4.12) для малых $\beta_{2}$, больших $N_{2}$ и некоторого $q$ справедливы следующие неравенства:

$$
0<q+3 \beta_{2}+3 \beta_{2} q+m \varrho_{s}^{-2} B_{s}^{-1} Q_{0}^{-1} \leq q_{1}<|Q| Q_{0}^{-1} .
$$

Следовательно, для некоторого положительного $\omega_{1}$ выполняется неравенство

$$
\left|\sum^{\prime} e_{j} B_{j} \varrho_{j}^{2}+m\right| \geq \omega_{1}\left|B_{s}\right| \varrho_{s}^{2} .
$$

Для случая, когда все заряженные частицы имеют заряды одного знака, мы получаем сразу

$$
\left|\sum^{\prime}{ }^{\prime} e_{j} B_{j} \varrho_{j}^{2}+m\right| \geq \omega_{2}\left|B_{s}\right| \varrho_{s}^{2}
$$

где $\omega_{2}=\left|e_{s}\right| 2^{-1},\left|e_{s}\right|=\min _{j}\left|e_{j}\right|, e_{j} \neq 0$ и $N_{2}$ велико.

Далее,

$$
\sum_{j=1}^{n} k_{j} \rho_{j}^{2}=\rho_{s}^{2} \sum_{j=1}^{n} k_{j} \rho_{j}^{2} \rho_{s}^{-2} \leq 2 M \rho_{s}^{2} .
$$

Подставляя $(4.16),(4.17)$ в (4.9), получаем

$$
F_{m_{1}, \ldots, m_{n}}(\varrho) \geq \omega^{2}\left|B_{s}\right|^{2} \varrho_{s}^{2},
$$

где $\omega^{2}=\min \left\{\omega_{1}^{2}, \omega_{2}^{2}\right\}(2 M)^{-1}$.

В силу (4.6), (4.7)

$$
(A \varphi, \varphi) \geq \sum(m) \omega^{2}\left(\left|B_{s}\right|^{2} \varrho_{s}^{2} f_{m_{1}, \ldots, m_{n}}, f_{m_{1}, \ldots, m_{n}}\right)(2 \pi)^{n}=\omega^{2}\left(\left|B_{s}\right|^{2} \varrho_{s}^{2} \varphi, \varphi\right) .
$$

Вследствие (4.12), (2.5) $\left|B_{s}\right| \varrho_{s} \rightarrow \infty$, если $|\varrho|=|\rho| \rightarrow \infty$. Т.к. $\varphi(\rho)=\varphi_{t}(\rho)$ и в силу (4.2) соотношение (4.1) доказано.

Автор хочет искренне поблагодарить проф. И. Сигала и Д. Колемана за приглашение в университеты Торонто и Кингстона (Канада), где эта работа была задумана, и проф. Т.Хоффманн-Остенхоффа за приглашение в Международный институт математической физики им. Эрвина Шредингера, где эта работа была сделана.

Поддержано Федеральным министерством науки и исследований (Австрия), Международным научным фондом (грант № R 94000) и Российским фондом фундаментальных исследований (грант № 94-01-01376). 


\section{Список литературы}

[1] С.А.Вугальтер, Г.М.ЖКислин // ТМФ. 1993. Т. 97. №1. С. 94-112.

[2] G.Zhislin Proc. of Euler International Math. Inst., St.-Petersburg (in print).

[3] J.E.Avron, J.W.Herbst, B.Simon // Ann. of Phys. 1978. V. 114. P. 431.

[4] С.А.Вугальтер, Г.М.ЖКислин // ДАН СССР. 1991. Т. 317. С. 1365.

[5] С.А.Вугальтер, Г.М.Жислин Препринт НИРФИ № 320. Н. Новгород, 1991.

[6] S.A.Vugalter // Commun. Math. Phys. (in print).

[7] М.А.Антонеи, Г.М.Жислин, И.А.Шерешевский Дополнение к русскому переводу книги: К.Йоргенс, Г.Вайдманн. Спектральные свойства гамильтоновых операторов. М.: Мир, 1973. [8] S.A.Vugalter, G.M.Zhislin // ROMP. 1984. V. 19. P. 39.

Научно-исследовательский радиофизический институт,

Поступила в редакцию Нижний Новгород

\section{G. M. Zhislin}

LOCATION OF THE ESSENTIAL SPECTRUM OF

THE ENERGY OPERATORS OF THE QUANTUM SYSTEMS WITH NONINCREASING MAGNETIC FIELD

The theorem on the localization of the essential spectrum is proved for nonneutral many-particle systems in nonincreasing magnetic fields having $S O(2)$ symmetry. 